\title{
Modelo de políticas contables para la toma de decisiones en las pequeñas y medianas empresas
}

\author{
Accounting policy model for decision-making in small and medium-sized companies
}

\section{Silvia Eugenia Lira Molina}

Artículo recibido en diciembre 2020

Arbitrado en febrero 2021

Aceptado en febrero 2021

Publicado en mayo 2021

sil17lm@gmail.com

ORCID: 0000-0001-5712-1768

Universidad Andina Simón Bolívar

Sucre - Bolivia

RESUMEN | El objetivo del artículo fue analizar las prácticas contables aplicadas en las pequeñas y medianas empresas (PYMES), para proponer un modelo de políticas contables que contribuya a la toma de decisiones. Se tomó como objeto de estudio a las PYMES de la ciudad de Sucre-Bolivia; se aplicaron encuestas a 373 empresas seleccionadas mediante muestreo probabilístico aleatorio simple, se estudiaron los estados financieros del $74 \%$ de esta muestra. Se aplicó el método Delphi para la validación del modelo.

Como resultado se sabe que son pocas las empresas que implementaron políticas contables orientadas al crecimiento empresarial; si bien no hacen planificación financiera y los informes contables no son usados con visión estratégica, los representantes reconocen su utilidad. Por tanto, se propuso un modelo de políticas contables tendiente a contribuir a la toma de decisiones. Se concluyó que las prácticas, políticas y procedimientos contables son adecuados para proveer información y recomendaciones en la toma de decisiones.

Palabras clave:

Políticas; Toma de decisiones; Estrategia; Empresas

\begin{abstract}
The objective of the article was to analyze the accounting practices applied in SMEs, to propose a model of accounting policies that contributes to decision-making. The SMEs of Sucre-Bolivia were taken as an object of study; Surveys were applied to 373 companies selected by simple random sampling, the financial statements of $74 \%$ of this sample were studied. Delphi method was applied for the validation of the model. As a result, it is known that few companies have implemented accounting policies aimed at business growth; Although they do not do financial planning and accounting reports are not used with strategic vision, the representatives recognize their usefulness. Therefore, an accounting policy model was proposed to contribute to decision-making. It was concluded that accounting practices, policies and procedures are adequate to provide information and recommendations for business strategy.

Keywords:

Accounting; Policies; Decision making; Strategy; Companies
\end{abstract}




\section{INTRODUCCIÓN}

En Bolivia, la actividad de las pequeñas y medianas empresas (PYMES) desempeña un papel importante en la generación de empleo e ingresos, sobre todo en manufactura, comercio y servicios (Collao, 2011); sin embargo, los márgenes de rentabilidad no son óptimos. Existen ciertas situaciones que pueden generar problemas en el manejo contable de estas empresas, afectando de manera directa o indirectamente a la toma de decisiones. Estas decisiones están sujetas a políticas contables que, según Catacora (1998) son los principios, bases, acuerdos, reglas y procedimientos asumidos por las organizaciones en la elaboración de estados financieros; noción aceptada en la actualidad (Van Horne, 2012). Existe entonces una relación entre la información y la toma de decisiones en la que la combinación de personas, registro y procedimientos se refleja en las políticas contables.

Las políticas contables determinan su influencia en el marco de la normativa contable y de la estandarización de documentación que surge como efecto de la implementación de esta normativa (Gómez, 2018). La Normas Internacionales de Información Financiera (NIIF) permite presentar estados financieros confiables, razonables y comprensibles, mejorando con esto la calidad de la información contable (Asociación Interamericana de Contabilidad, 2014). Si bien existe una normativa específica para PYMES: NIIF para PYMES, su aplicación no es habitual en Bolivia, a pesar de que la tendencia internacional así lo sugiere (Morales, 2010).

Existe una falencia en la concepción asumida por varias empresas acerca de la importancia de las políticas y procedimientos contables, pues las prácticas actuales se basan más en la costumbre, la repetición y la imitación, que en verdaderos conocimientos de los principios contables. Adicionalmente, poco uso se hace del criterio profesional y su posterior injerencia en el proceso de la toma de decisiones (Rondón, 2008). En muchos casos, la contabilidad aplicada en las PYMES de Bolivia poco aporta a la toma de decisiones.

Los empresarios de estas empresas priorizan los objetivos operativos, descuidando el establecimiento de un plan estratégico. Ante la ausencia de este direccionador, el empresario se constituye en un administrador reactivo que actúa de acuerdo con los imperativos que le impone su entorno competitivo (Zurita, 2015).

Esta investigación tuvo por objetivo analizar las prácticas contables aplicadas en las PYMES en la ciudad de Sucre, para proponer un modelo de políticas contables que contribuya a la toma de decisiones de las empresas. Con este propósito los apartes del documento son: descripción del panorama general; consulta a las empresas; revisión de los estados financieros; propuesta de un modelo de políticas contables.

\section{METODOLOGÍA}

Se trata de una investigación de corte transversal que, según la etapa, tuvo alcance descriptivo o relacional; requirió de la unión de los enfoques cuantitativo y cualitativo para alcanzar el objetivo planteado; está dividida en cuatro partes.

La primera parte permitió desde un enfoque cualitativo describir el panorama general de las PYMES en Sucre mediante recopilación de información secundaria y terciaria. Los métodos aplicados fueron análisis histórico lógico e inductivo-deductivo. 
La segunda etapa se enfocó en consultar a las empresas sobre el uso y percepción de valor de las políticas y prácticas de contabilidad. Se usó la técnica de la encuesta para recoger información primaria de las empresas. El diseño del instrumento permitió dar tratamiento cualitativo y cuantitativo a las variables de interés; se contó con validación interna del instrumento gracias al criterio de ocho expertos en estrategia empresarial y consultoría contable para PYMES. La definición del tamaño de muestra consideró el registro de FUNDEMPRESA, que informa que se tiene un total de 11998 PYMES registradas en Sucre de diversos rubros $y$ actividades (universo poblacional para este estudio). Se aplicó el muestreo probabilístico aleatorio simple, con base en la siguiente fórmula.

$$
n=\frac{Z^{2} * P * q * N}{e^{2}(N-1)+z^{2} * P * q}
$$

Donde se considera: $\mathrm{e}=5 \%$ error; $\mathrm{z}=1,96$ (corresponde al $95 \%$ de margen de confiabilidad); N= 11998 empresas (universo poblacional); $p=50 \%$ de probabilidad de éxito; $q=50 \%$ de probabilidad de fracaso y $n$ es el tamaño de la muestra a definir.

$$
n=\frac{1,96^{2} * 0.50 * 0.50 * 11998}{0.05^{2}(11998-1)+1,96^{2} * 0.50 * 0,50}
$$

\section{n: $\mathbf{3 7 3}$}

Según el resultado obtenido, se trabajó con una muestra representativa de 373 PYMES del Sucre.

La tercera parte, consistió en la revisión de los estados financieros de 216 PYMES (equivalente al $74 \%$ del total de la muestra establecida), dado que el acceso a la información fue reducido en la mayoría de las empresas, se tuvo que dar un enfoque cualitativo a esta fase, logrando como resultado describir las principales característica, coincidencias o contradicciones en relación con los resultados obtenidos en la encuesta previa.

Habiendo determinado las condiciones básicas que las políticas contables deben cumplir para solucionar los problemas que las PYMES, en la cuarta y última parte de los resultados coma se propuso un modelo de políticas contables. Para validad el modelo se aplicó el método Delphi (García \& Suárez, 2013), consultando a un grupo de ocho expertos en estrategia empresarial y consultoría contable para PYMES.

\section{RESULTADOS}

Se presenta un diagnóstico de las PYMES de Sucre, en el que se emplearon las técnicas de encuesta, observación y análisis histórico lógico (de estados financieros), para conocer la existencia de políticas contables y su modo de aplicación. Asimismo, se determinaron las necesidades básicas que dichas políticas deben cumplir para solucionar problemas relacionados a la toma de decisiones.

\section{Panorama general}

La alta proporción de actividad informal en las PYPES es un problema presente a nivel nacional y específicamente en Sucre. El incremento de la productividad se constituye en un reto para promover su inclusión a la economía formal. La informalidad se atribuye a carga normativa, carga tributaria y desconocimiento de los beneficios de la formalización. 
Las políticas contables pueden definirse dentro de los siguientes parámetros: ayuda financiera, asesoramiento, trámites, capacitación, vinculaciones, control de aspectos tributarios, proyecciones a futuro.

Los problemas de las PYMES de Sucre relacionados con contabilidad se pueden sintetizar en: deficiencias en planes estratégicos; estructuras de costeo inadecuadas; desaprovechamiento de políticas de promoción; desconocimiento de normativas (nacional/internacional); valoración selectiva con base a política comercial/industrial del momento; incumplimientos de normas de calidad; desconocimiento del mercado; contabilidad pensada únicamente en cumplir obligaciones fiscales; desaprovechamiento de líneas de crédito para PYMES; desconocimiento del mercado (cliente, competencia, proveedor); bajo poder de negociación; deficiente selección de canales de distribución; bajo control de producción; poco desarrollo tecnológico; modelos de administración familiar; prácticas inadecuadas en selección y contratación de personal.

Las fortalezas que se pueden derivar del ámbito de la contabilidad son: normativa que favorezca la contabilidad estratégica; previsiones financieras; planificación y proyecciones; valoración de los alcances y usos de la información contable; previsiones de gastos (trámites, capacitaciones, etc.); controles de costos de producción y gastos relacionados; adecuación a normativa laboral; control; previsión de costos de mercado y adecuación a la competencia; control de pasivos $y$ proyecciones de endeudamiento.

\section{Consulta a las empresas}

Respecto a los aspectos normativos más problemáticos y costosos, el $43,62 \%$ de las PYMES considera que son los impuestos; el $31,17 \%$ las tasas y patentes; el $21,45 \%$ los trámites de constitución (Fundempresa y registros legales); y 3,76\% pagos imprevistos.

El $77,75 \%$ de las empresas no realiza previsiones de manejo económico aparte de las establecidas por ley, el 22,25\% sí lo hace. Asimismo, del total de las empresas, indiferentemente de si realiza previsiones: el $47,99 \%$ considera que hacer inversiones es el principal beneficio de la formalización, el 21,98\% considera que es el acceso a seguros médicos, el $17 \%$ resalta la capacidad de ahorrar en el corto plazo y el $2,95 \%$ considera q el ahorro a largo plazo es la principal ventaja.

Por otra parte, $24,13 \%$ de estas empresas no planifica, el $46,11 \%$ planifica como máximo a un año, el 25,47\% planifica en un horizonte de tres años y sólo el $4.29 \%$ planifica a más de 3 años.

La Tabla 1 presenta los resultados obtenidos respecto frecuencia de proyecciones de ingresos y egresos, revisión del balance general y los estados de resultados. 


\section{Tabla 1}

Frecuencia de análisis y consulta a los estados financieros

\begin{tabular}{lccc}
\hline \multicolumn{1}{c}{ Frecuencia } & $\begin{array}{c}\text { Proyección de } \\
\text { ingresos y egresos } \\
\text { En \% }\end{array}$ & $\begin{array}{c}\text { Consulta a Balance } \\
\text { General } \\
\text { En \% }\end{array}$ & $\begin{array}{c}\text { Consulta a Estados } \\
\text { de Resultado } \\
\text { En \% }\end{array}$ \\
\hline varias veces al mes & 13,40 & 17,96 & 0,80 \\
varias veces en 3 meses & 46,11 & 2,95 & 8,58 \\
una vez cada 3 meses & 14,75 & 31,64 & 16,89 \\
una vez cada 6 meses & 1,61 & 30,83 & 23,06 \\
al final de la gestión & 24,13 & 16,62 & 50,67 \\
\hline \multicolumn{1}{c}{ Total } & $\mathbf{1 0 0 , 0 0}$ & $\mathbf{1 0 0 , 0 0}$ & $\mathbf{1 0 0 , 0 0}$ \\
\hline
\end{tabular}

Según se observa en la Tabla 1, el 59,51\% de las empresas hace proyecciones financieras por lo menos una vez en tres meses, las proyecciones son consideradas solamente una vez por gestión en el $24,13 \%$ de las empresas. La revisión del balance general se hace varias veces al mes en el $17,56 \%$ de las empresas y más de la mitad lo hace por lo menos una vez cada tres meses. Respecto a la consulta de los estados de resultados, el 50\% de las empresas los analiza sólo al final de la gestión.

\section{Tabla 2}

Previsiones de gastos o reserva dinero, control de gastos estudio de los cambios en el mercado

\begin{tabular}{ccccc}
\hline Frecuencia & $\begin{array}{c}\text { Proyección de } \\
\text { ingresos y egresos } \\
\text { en } \%\end{array}$ & $\begin{array}{c}\text { Consulta a } \\
\text { Balance } \\
\text { General } \\
\text { en } \%\end{array}$ & $\begin{array}{c}\text { Consulta a Estados } \\
\text { de Resultado } \\
\text { en } \%\end{array}$ & $\begin{array}{c}\text { Importancia de la } \\
\text { previsión } \\
\text { en } \%\end{array}$ \\
\hline Si & 6,17 & 29,22 & 1,07 & 100 \\
No & 93,83 & 70,78 & 98,93 & 0 \\
\hline Total & $\mathbf{1 0 0 , 0 0}$ & $\mathbf{1 0 0 , 0 0}$ & $\mathbf{1 0 0 , 0 0}$ & $\mathbf{1 0 0 , 0 0}$ \\
\hline
\end{tabular}

Según se muestra en la Tabla 2, el 93,83\% de las PYMES no realiza previsiones de gastos 0 reserva dinero para gastos imprevistos. Asimismo, el 70,78\% de estas empresas no realiza un control constante en gastos de producción o de servicios. Sólo el 1,07\% realiza previsiones respecto a cambios en la demanda, costos de insumos y competencia. Finalmente, a pesar de los resultados anteriores, llama la atención que el $100 \%$ de las empresas objeto de estudio, requiere de una planificación o previsión en el manejo de pasivos, deudas y apalancamientos.

Los representantes de las empresas expresaron lo siguiente: existen problemas con impuestos y con pagos imprevistos como el doble aguinaldo; con relación al control de planillas existen dificultades con la retención y los pagos de los beneficios sociales, especialmente cuando no existe una conciliación entre el empleador y el empleado; las proyecciones son escasas 0 no son 
consideradas como parte integrante del desarrollo del trabajo en las empresas; no se obtienen beneficios al analizar los estados financieros.

\section{Revisión de los estados financieros}

Se realizaron pruebas a través del análisis documental de los estados financieros a los que se tuvo acceso (un total de 216 estados financieros, equivalente al $74 \%$ del total de la muestra establecida). Las evaluaciones se realizaron mediante la comparación directa del balance, los estados de resultados y la valoración de las cuentas según el plan estandarizado de las respectivas cuentas. Lamentablemente, en algunos casos las empresas permitieron acceso limitado, en cambio en otros se logró hacer un análisis detallado.

A continuación, se presenta una síntesis de los resultados: (a) No existen variaciones considerables entre la valoración de los estados financieros y el resultado de la consulta a los representantes de las empresas, lo que corrobora la certeza de lo expresado en la encuesta. (b) El concepto de políticas contables no es realmente común, se aplica el concepto de normas nacionales y adecuaciones, según lo requerido. En realidad, no existen políticas contables per se, sino se definen prácticas contables según lo que se cree conveniente y las mismas se cambian en el caso de presentarse algún conflicto. En este sentido, ninguna de las empresas evaluadas tiene un modelo de políticas contables que se haya formulado con base en los objetivos, misión, visión o necesidades de la empresa. (c) Se debería tener una evaluación de las normas contables y su adecuación a las mismas para determinar las prácticas contables que son requeridas dentro de la empresa, según el rubro o actividad pertinente y que, también, vaya contribuyendo al desarrollo de su crecimiento. (d) No se tienen realmente previsiones sino las consideradas como determinadas por ley o generales (indemnizaciones relacionadas con el finiquito, por ejemplo). (e) No se usa la información contable dentro de un proceso de decisión.

Para el diseño de políticas contables se deberá considerar las aclaraciones siguientes.

\section{Nota a los estados financieros sobre políticas contables}

La nota de políticas contables debe incluir la revelación de estas, para todos los rubros cuya acumulación haya sido importante, o cuyos saldos en cualquiera de los dos periodos, sean significativos dentro del rubro y dentro de los totales.

No existen notas que refieran, directamente, la existencia o aplicación de alguna política contable definida. Esta política refiere aspectos como la naturaleza de sus operaciones y sus principales actividades: el domicilio legal incluyendo el correspondiente a sus áreas de operación o localización de facilidades; su forma legal, incluyendo disposiciones de ley pertinentes a su creación o funcionamiento; la indicación de ser una subsidiaria; otra información breve sobre cambios fundamentales como incrementos o disminuciones en su capacidad productiva, área de operaciones, entre otros.

\section{Políticas relacionadas con moneda extranjera}

Aparte del ajuste por redondeo, y debería tener una reexpresión de los saldos de pago o cobro a la fecha de los estados financieros, las ganancias o pérdidas por diferencia en cambio, deberían registrarse en cuentas de resultados. La diferencia de cambio neta debería revelarse en el estado de resultados. 


\section{Cuentas por cobrar y provisión de cuentas de dudosa cobranza}

Las cuentas por cobrar se deben clasificar en una de las cuatro categorías de instrumentos financieros, según lo expuesto por la Norma Internacional de Contabilidad No39 (NIC 39) (Monge, 2005). Además, se debe reconocer un menor valor si el importe en libros es mayor que su valor recuperable estimado. Para ello, la empresa debe evaluar en cada fecha de reporte si existe evidencia de que el activo ha perdido valor. La fecha de reporte es realizada directamente a fin de gestión.

$\mathrm{Se}$ debe distinguir las operaciones realizadas con empresas del estado y entidades del sector público de aquellas realizadas con terceros, mostrando el movimiento que han tenido en el año. No se encontró diferencia en lo evaluado. Sin embargo, se encontraron mecanismos de control de riesgo en 56 empresas.

\section{Control de existencias}

Más de una vez al año debería tenerse una conciliación de los saldos en libros con los respectivos inventarios físicos. El control de inventario se hace con inventario inicial y final directamente, ya que esto es obligatorio. Las existencias deben ser valuadas al menor valor entre: costo de existencias o valor neto de realización; aspecto que no se aplica actualmente.

\section{Inmuebles y equipos}

Conciliación realizada una vez por gestión. Estos activos se valúan al costo 0 valor revaluado (tratamiento alternativo permitido) menos pérdidas por desvalorización, menos depreciación acumulada. No se consideran reparaciones o mantenimientos, sino gastos.

\section{Diferencia de cambio como gasto}

No debería ser reconocida como costo, lo cual sucede en algunas empresas. Esto afecta a la valoración en general.

\section{Intangibles}

Se debería reconocer como intangibles aquellos activos adquiridos $\circ$ generados internamente y que producen beneficio económico futuro. El costo del activo puede ser medido fiablemente. Esta no es una práctica común, ya que no se manejan en general intangibles.

Técnicamente es posible completar la producción del activo intangible, de forma tal que esté disponible para su uso o venta. Se requiere: la intención expresa de la entidad de completar el activo referido; demostrar su capacidad de utilizar o vender; demostrar la existencia de un mercado para la producción que genere el activo intangible; disponibilidad de recursos técnicos, financieros o de otro tipo para completar el desarrollo y para usar o vender; y capacidad para medir fiablemente el desembolso atribuible al activo intangible durante su desarrollo.

\section{Pérdidas por deterioro}

La entidad debería reconocer el menor valor de sus activos a través de alguno de los siguientes indicios: disminución significativa del valor de mercado; cambios significativos adversos para la empresa en el entorno tecnológico, comercial, económico o legal en que opera o en el cual está dirigido el activo; evidencia sobre la obsolescencia o daño físico del activo; cambios significativos con efecto adverso en relación con el grado o forma en que el activo se usa o se espera usar; y evidencia de que rendimiento económico del activo será 
inferior al esperado. Dentro de lo evaluado, las empresas se limitan a considerar la depreciación.

\section{Compensaciones, previsiones indemnizaciones, previsiones imprevistos laborales} para

\section{para}

Existente en empresas constructoras y algunas empresas agrícolas. Se tiene en general una previsión para indemnizaciones, pero esta surge usualmente cuando es requerida, es decir, no se hace con base a planillas.

\section{Ingresos diferidos}

Los ingresos diferidos no son considerados en la mayoría de estas empresas. Esto es problemático cuando se trata de la venta de productos con pagos diferidos o "fiados", ya que generan un desbalance en el inventario.

La prudencia exige que, si no existe expectativa de un ingreso de beneficios económicos a la entidad, no se reconocen ni los ingresos ni los márgenes de ganancias; sin embargo, si se espera la recepción del beneficio económico, éste debería incluir el margen de ganancia o utilidad, que no se considera para una proyección.

\section{Débito y crédito fiscal}

No se tiene una relación general entre estas cuentas, ni se tiene una referencia notoria del saldo a favor, las empresas suponen que es difícil de generar.

\section{Contingencias}

Se debería tener cuentas para diferentes aspectos laborales, de producción o imprevistos, aduciendo de paso los efectos financieros probables. Esto genera un problema al momento de reaccionar o ver la incidencia de cualquier evento, como es el caso de los movimientos sociales.

\section{Modelo para la implementación de políticas contables}

Es conveniente resaltar que esta propuesta tiene un prototipo base evaluado por expertos en la primera formulación del método Delphi, para verificar la necesidad o pertinencia de los aspectos seleccionados como comunes para todas las empresas y que pueden ser extendidos o considerados generales.

El diseño por desarrollar deberá tener la estructura presentada en la Figura 1.

\section{Figura 1}

Estructura modelo para el diseño de políticas contables

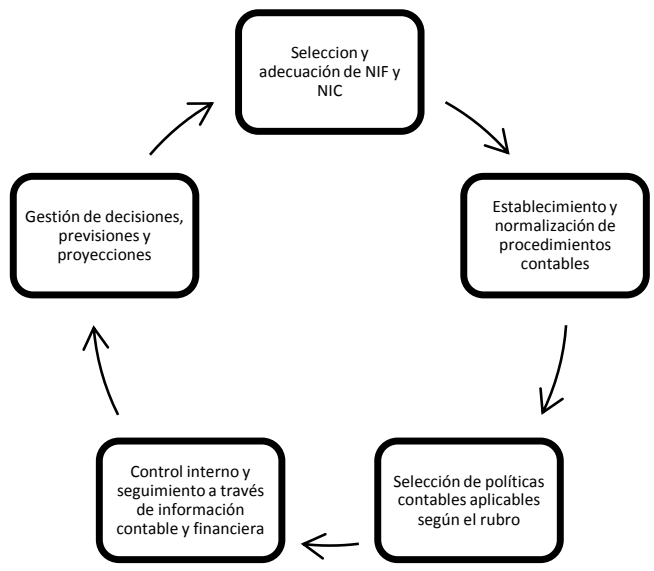


Siguiendo la estructura propuesta en la Figura 1, se tiene un modelo de procedimiento que contribuye a la aplicación de políticas contables orientadas a contribuir a la toma decisiones, aspectos que no se consideran en la actualidad, ya que no existen proyecciones, prevenciones 0 previsiones que puedan establecer cambios y/o inversiones a futuro.

Dado que estas políticas se deben adecuar a la normativa nacional, el modelo de políticas deberá reflejar lo necesario para todas las empresas, ya que cualquiera de ellas podrá seleccionar las que considere pertinentes y adaptarlas de acuerdo con sus condiciones y requerimientos. A continuación, se desglosan los cinco componentes descritos en la Figura 1.

\section{Selección y adecuación de NIF y NIC}

Las normas que deberían considerarse (excluyendo aquellas que están sujetas a evaluación), según la Resolución N N. 02/2010 emitida por el CTNAC, homologada por CAUB, desarrolladas en convergencia con las NIIF (Mantilla, 2013), son las siguientes.

Normas de Información Financiera - NIF (NIF, 2011): NIF-1, adopción por primera vez de las normas de información financiera; NIF-2, pagos basados en acciones; NIF-3, combinaciones de negocios; NIF-4, contratos de seguros; NIF-5, activos no corrientes mantenidos para la venta y operaciones discontinuadas; NIF-6, exploración y evaluación de recursos naturales; NIF-7, instrumentos financieros: Información a revelar; y NIF-8, segmentos de operación.

Normas de Contabilidad - NC: NC-1, presentación de estados financieros; NC-2, inventarios; NC-7, estados de flujos de efectivo; NC-8, políticas contables, cambios en las estimaciones contables y errores; NC-10, hechos ocurridos después de la fecha del balance; NC11 , contratos de construcción; NC-12, impuestos a las ganancias; NC-16, propiedad, planta $y$ equipo; NC-17, arrendamientos; NC-18, ingresos de actividades ordinarias; NC-19, Beneficios a los empleados; NC-20, contabilización de las subvenciones del gobierno e información a revelar sobre ayudas gubernamentales; NC-21, efectos de las variaciones en las tasas de cambio de la moneda extranjera; NC-23, costos por préstamos; NC-24, información a revelar sobre partes relacionadas; NC-26, contabilización e información financiera sobre planes de beneficio por retiro; NC-27, estados financieros consolidados y separados; NC-28, inversiones en empresas asociadas; NC-29, financiera en economías hiperinflacionarias; NC-31, participaciones en negocios conjuntos; NC-32, instrumentos financieros: presentación e información a revelar; NC-33, ganancia a revelar; NC-34, información financiera intermedia; NC36, deterioro del valor de los activos; NC-37, provisiones, activos contingentes y pasivos contingentes; NC-38, activos intangibles; NC-39, instrumentos financieros: reconocimiento $y$ medición; NC-40, propiedad de inversión; NC41, agricultura.

Estas normas son las que determinan los procedimientos contables a ser aplicados y que deben definirse para poder procedimientos contables.

\section{Establecimiento y normalización de procedimientos contables}

La contabilidad de cada PYME debe ser suficientemente detallada para permitir el registro de todas las operaciones, conforme a las normas determinadas, así como para la preparación de la información financiera y económica establecida para la actividad o rubro. 
Ésta debe ser establecida inicialmente sin que exista determinación de lo contario, ya que la falencia de esta estandarización o normalización determina la aplicación de las políticas posteriores.

Dentro de este proceso se debe considerar los siguientes componentes.

Documentos comerciales, como constancia y base del registro contable de las transacciones comerciales $y / o$ financiera realizadas por PYMES, lo primero es definir los comprobantes internos emitidos en la empresa que pueden entregarse a terceros y los que pueden circular en la misma empresa. Ej.: facturas de ventas, recibos por cobranzas, presupuestos y vales. Por otra parte, se deben registrar documentos comerciales, instrumentos públicos (escrituras, hipotecas) y privados (contratos de arrendamiento, depósito) y cualquier otro documento que sea respaldo de una registración contable o que es fuente de información (planillas de sueldos, informes de pagos).

Los comprobantes externos son aquellos emitidos fuera de la empresa y luego recibidos y conservados en la empresa, deben ser definidos para determinar la política respectiva. Por ejemplo, no es la misma aplicación para recepción de facturas que para la recepción de recibos, teniendo que efectuar la correspondiente retención impositiva, y en el caso dado, determinar para qué tipos de productos o servicios se reciben, dado que no se debería considerar todo en no deducibles.

Libros contables, documentos que deben ser evaluados y están definidos por normativa. Deben ser evaluados y conformados para evitar problemas posteriores. Se tiene la tendencia a evitar estos fundamentos, como por ejemplo no tener notariados el Libro Diario o el LCV. Todos las PYMES deben conformar su contabilidad en libros y registros contables de acuerdo con la normatividad del código del comercio y las disposiciones legales.

Al iniciar la actividad comercial, la empresa debe elaborar un inventario y un balance general que le permitan conocer de manera clara y completa la situación de su patrimonio, en este libro deben registrarse todos estos rubros, de acuerdo con las normas legales. Lo recomendable es: libro de compra venta (LCV), libro diario, libros mayores, kárdex de inventario físico y valorado y libros auxiliares que sean necesarios (como libro de control de caja o seguimiento de pagos). Asimismo, realizar el correspondiente control y mantenimiento de facturas, tanto entrantes como salientes.

\section{Selección de políticas contables aplicables según el rubro}

Las políticas contables sugeridas y estandarizadas dentro del modelo, deben ser adecuadas, aplicadas o eliminadas según la necesidad de la empresa considerando las necesarias específicas dentro de la aplicación de las PYMES.

\section{Control interno y seguimiento a través de información contable y financiera}

El modelo que se propone permite realizar control interno y externo, los estados financieros se refieren siempre a un ente donde el propietario es considerado como tercero, por tanto, es sujeto a control como todos los otros participantes.

Bienes económicos, los estados financieros se refieren siempre a bienes económicos, es decir bienes materiales e inmateriales que posean valor económico y por ende susceptible de ser valuados en términos monetarios. 
Moneda común denominador, los estados financieros reflejan el patrimonio mediante un recurso que se emplea para reducir todos sus componentes heterogéneos a una expresión que permita agruparlos y compararlos fácilmente. Este recurso consiste en elegir una moneda de cuenta y valorizar los elementos patrimoniales aplicando un precio a cada unidad. Generalmente se utiliza como moneda de cuenta el dinero que tiene curso legal en el país dentro del cual funciona el ente y en este caso el precio está dado en unidades de dinero de curso legal.

Empresa en marcha o negocio en marcha, salvo indicación expresa, se entiende que los estados financieros pertenecen a una empresa en marcha, considerándose que el concepto que informa la mencionada expresión se refiere a todo organismo económico cuya existencia temporal tiene plena vigencia y proyección futura.

Valuación al costo 0 valor histórico original, el valor de costo - adquisición o producción- constituye el criterio principal y básico de valuación, que condiciona la formulación de los estados financieros conocidos también con el concepto de empresa en marcha, razón por la cual esta norma adquiere el carácter de principio. Esta afirmación no significa desconocer la existencia y procedencia de otras reglas y criterios aplicables en determinadas circunstancias, sino afirmar que en caso de no existir una circunstancia especial que justifique la aplicación de otro criterio, debe prevalecer el de costo como concepto básico de valuación.

Es una condición que los ejercicios sean de igual duración, para que los resultados de dos o más ejercicios sean comparables entre sí. Sin embargo, las empresas con fines administrativos y financieros pueden medir sus estados financieros anual, semestral, cuatrimestral, bimestral, etc.

Devengado, las variaciones patrimoniales que deben considerarse para establecer el resultado económico son las que competen a un ejercicio sin entrar a considerar si se han cobrado o pagado.

Objetividad o confiabilidad, los cambios en los activos, pasivos y en la expresión contable del patrimonio neto deben reconocerse formalmente en los registros contables, tan pronto como sea posible medirlos objetivamente y expresar esa medida en moneda de cuenta.

Realización, los resultados económicos sólo deben computarse cuando sean realizados, o sea cuando la operación que los origina queda perfeccionada desde el punto de vista de la legislación o prácticas comerciales aplicables y se hayan ponderado fundamentalmente todos los riesgos inherentes a tal operación. Debe establecerse con carácter general que el concepto realizado participa del concepto devengado.

Prudencia o criterio conservador, significa que cuando se deba elegir entre dos valores para un elemento del activo, normalmente se debe optar por el más bajo, o bien que una operación se contabilice de tal modo que la alícuota del propietario sea menor. Este principio general se puede expresar también diciendo: 'contabilizar todas las pérdidas cuando se conocen y las ganancias solamente cuando se hayan realizado'.

Uniformidad o consistencia, los principios generales, cuando fueren aplicables, y las normas particulares utilizados para preparar los estados financieros de un determinado ente deben ser aplicados uniformemente de un 
ejercicio a otro. Debe señalarse por medio de una nota aclaratoria, el efecto en los estados financieros de cualquier cambio de importancia en la aplicación de los principios generales y de las normas particulares.

\section{Significatividad,}

materialidad

$\mathbf{0}$ importancia relativa, al ponderar la correcta aplicación de los principios generales y normas particulares debe necesariamente actuarse con sentido práctico. Frecuentemente se presentan situaciones que no encuadran dentro de aquellos y que, sin embargo, no presentan problemas porque el efecto que producen no distorsiona el cuadro general. Desde luego, no existe una línea demarcatoria que fije los límites de lo que es y no es significativo $y$ debe aplicarse el mejor criterio para resolver lo que corresponda en cada caso, de acuerdo con las circunstancias, teniendo en cuenta factores tales como el efecto en los activos o pasivos, en el patrimonio o en el resultado de las operaciones.

Exposición, los estados financieros deben contener toda la información y discriminación básica y adicional que sea necesaria para una adecuada interpretación de la situación financiera y de los resultados económicos del ente a que se refieren.

\section{Gestión de decisiones, previsiones y proyecciones}

Si bien, como ya se a argumentado, cada empresa deberá analizar y adecuar los criterios que se ajustan a sus necesidades. En la Tabla 3 se presenta una categorización de la pertinencia de aplicación del modelo en la toma de decisiones, procedimientos de auditoría, prácticas contables y políticas contables.

\section{Tabla 3}

Estructura modelo para el diseño de políticas contables

\begin{tabular}{|c|c|c|c|}
\hline Variable & Dimensión & Indicador & Valor \\
\hline \multirow[t]{3}{*}{$\begin{array}{l}\text { Toma de } \\
\text { decisiones }\end{array}$} & Prácticas contables & $\begin{array}{l}\text { Prácticas contables orientadas al desarrollo del } \\
\text { trabajo }\end{array}$ & $\begin{array}{l}\text { Muy } \\
\text { adecuado }\end{array}$ \\
\hline & $\begin{array}{l}\text { Políticas contables } \\
\text { establecidas }\end{array}$ & $\begin{array}{l}\text { Procesos establecidos para el funcionamiento de } \\
\text { las políticas contables }\end{array}$ & $\begin{array}{l}\text { Muy } \\
\text { adecuado }\end{array}$ \\
\hline & $\begin{array}{l}\text { Procedimientos de } \\
\text { auditoría }\end{array}$ & Procesos de control interno & Adecuado \\
\hline \multirow[t]{2}{*}{$\begin{array}{l}\text { Procedimientos } \\
\text { de auditoría }\end{array}$} & $\begin{array}{l}\text { Procedimientos } \\
\text { comunes }\end{array}$ & $\begin{array}{l}\text { Procedimientos de auditoría que se realizan } \\
\text { constantemente }\end{array}$ & Adecuado \\
\hline & $\begin{array}{l}\text { Procedimientos } \\
\text { poco comunes }\end{array}$ & $\begin{array}{l}\text { Procedimientos de auditoría que no se aplican } \\
\text { generalmente pero que son necesarias para el } \\
\text { desarrollo de las funciones de las PYMES }\end{array}$ & Adecuado \\
\hline
\end{tabular}




\begin{tabular}{|c|c|c|c|}
\hline Variable & Dimensión & Indicador & Valor \\
\hline \multirow[t]{2}{*}{$\begin{array}{l}\text { Prácticas } \\
\text { contables }\end{array}$} & Prácticas comunes & $\begin{array}{l}\text { Prácticas contables que se realizan } \\
\text { constantemente }\end{array}$ & $\begin{array}{l}\text { Muy } \\
\text { adecuado }\end{array}$ \\
\hline & $\begin{array}{l}\text { Prácticas poco } \\
\text { comunes }\end{array}$ & $\begin{array}{l}\text { Prácticas contables que no se aplican } \\
\text { generalmente pero que son necesarias para el } \\
\text { desarrollo de las funciones de las PYMES }\end{array}$ & Adecuado \\
\hline \multirow[t]{2}{*}{$\begin{array}{l}\text { Políticas } \\
\text { contables }\end{array}$} & Políticas estándar & $\begin{array}{l}\text { Políticas necesarias de aplicación dentro de la } \\
\text { empresa }\end{array}$ & $\begin{array}{l}\text { Muy } \\
\text { adecuado }\end{array}$ \\
\hline & Políticas de elección & $\begin{array}{l}\text { Políticas que son flexibles para su aplicación } \\
\text { dentro de una empresa }\end{array}$ & $\begin{array}{l}\text { Muy } \\
\text { adecuado }\end{array}$ \\
\hline
\end{tabular}

Como se puede observar en la Tabla 3, en el contexto de las PYMES de la ciudad de Sucre, las prácticas y políticas contables son muy adecuadas para aportar a la toma de decisiones estratégicas, los procedimientos de auditoría son adecuados. Por otra parte, es adecuado implementar el modelo para favorecer los procedimientos de auditoría sean estos comunes o poco comunes. De manera similar, aplicar el modelo para prácticas comunes es muy adecuado y adecuado para prácticas poco comunes. Finalmente, de manera general es muy adecuado implementar el modelo para favorecer políticas estándar y de elección.

\section{CONCLUSIONES}

Se propuso un modelo de políticas contables adecuado para las PYMES, éste fue validado en el contexto de las empresas de la ciudad de Sucre. Se considera que las prácticas, políticas y procedimientos contables son adecuados para aportar a la toma de decisiones estratégicas. En Sucre son pocas las PYMES que han implementado políticas contables diseñadas $y$ orientadas a favorecer su crecimiento empresarial. La problemática identificada confirma que las empresas no hacen planificación financiera, los informes contables no son usados con visión estratégica y no se tiene una proyección de crecimiento.

En las PYMES que fueron estudiadas, no existen libros diarios uniformes, por la ausencia de un plan de cuentas que se identifique con cada una de las operaciones que se realiza en el ente económico. El no registrar un hecho económico de una empresa provoca el desconocimiento de las operaciones de manera cronológica, por lo tanto, no proporciona información clasificada para una correcta decisión; por lo que conlleva a pérdidas de oportunidades para la empresa. La información mal clasificada y registrada en cuentas que no corresponden llevará a efectuar estados financieros incorrectos, mismos que proporcionarán información inadecuada y decisiones desacertadas. Entender la utilidad de estas políticas permite efectuar proyecciones a largo plazo e identificar las debilidades tanto internas como externas, además de proporcionar información verídica, oportuna y confiable a los responsables para que puedan tomar decisiones acertadas.

Es necesario que todas las empresas tengan un plan de cuentas apropiado a sus actividades, 
que les permitan registrar de manera ordenada y clasificada las diferentes transacciones diarias. Es importante utilizar las herramientas de control, que permitan a la empresa conocer el flujo diario y los reportes correspondientes de las operaciones, para que el movimiento económico sea transparente y permita a los usuarios conocer la información, beneficiarse de la misma tomando decisiones en favor del crecimiento de la empresa.

\section{REFERENCIAS}

Asociación Interamericana de Contabilidad (2014) Importancia de la aplicación de la NIIF y para PYMES en Bolivia $y$ Latinoamérica. República dominicana: CIC

Catacora, F (1998) Contabilidad. La base para las decisiones gerenciales. Argentina: Astra

Collao, F. R. (2011). Políticas e instituciones de apoyo a las micro y pequeñas empresas en Bolivia. En: Apoyando a las pymes: políticas de fomento en América Latina y el Caribe. Santiago: CEPAL, 2011. p. 17-76. LC/R. 2180

García V, M. \& Suárez M, M. (2013). El método Delphi para la consulta a expertos en la investigación científica. Revista Cubana de Salud Pública, 39(2), 253-267
Gómez, G. (2018). Las normas internacionales de información financiera y la toma de decisiones financieras en las empresas. Quipukamayoc. 25. 73. 10.15381/quipu.v25i49.14282.Morales, A. M. C. (2010). Algunas experiencias exitosas en Latinoamérica relacionadas con la estandarización contable. Revista Facultad de Ciencias Económicas, 18(2), 207-226

Mantilla, S. A. (Ed.). (2013). Estándares/normas internacionales de información financiera (IFRS/NIIF). Ecoe Ediciones

Monge, P. (2005). Las normas internacionales de contabilidad. Actualidad contable FACES, 8(10), 35-52

NIF, C. (2011). Normas de Información Financiera

Rondón, F. (2008). Análisis de los estados financieros. Caracas: Fragor

Van Horne, J. (2012) Fundamentos de la administración financiera. México: Pearson Prentice Hall

Zurita, A. (2015) el Rol de las PYMES en el Mercosur. Argentina: Banco Interamericano de Desarrollo 